\title{
QM/MM Study of L-Lactate Oxidation by Flavocytochrome $b_{2}$
}

\author{
N. Gillet, ${ }^{\mathrm{a}, \mathrm{b}, \mathrm{c}}$ J. J. Ruiz-Pernia, ${ }^{\mathrm{b}}$ A. de la Lande, ${ }^{\mathrm{a}}$ B. Lévy, ${ }^{\mathrm{a}}$ F. Lederer, ${ }^{\mathrm{a}}$ I. Demachy, ${ }^{\mathrm{a}}$ V. Moliner ${ }^{\mathrm{b}}$ \\ [a]: Laboratoire de Chimie-Physique, CNRS UMR 8000, Université Paris Sud, Bâtiment 349 - Campus d'Orsay. 15, \\ avenue Jean Perrin,91405 Cedex Orsay, France. \\ [b]: Departament de Química Física i Analítica, Universitat Jaume I, 12071 Castellón, Spain. \\ [c]: present address: Department for Theoretical Chemical Biology, Institute for Physical Chemistry, Kaiserstrasse \\ 12, Karlsruhe Institute of Technology, 76131 Karlsruhe, Germany. \\ To whom correspondence should be addressed: natacha.gillet@kit.edu, moliner@uji.es
}

\section{$\underline{\text { Abstract }}$}

In this work, we have performed molecular dynamics simulations using a hybrid Quantum Mechanics/Molecular Mechanics (QM/MM) scheme to study the mechanism of L-lactate oxidation by flavocytochrome $b_{2}$ (Fcb2). Our results obtained at the QM(AM1)/MM level have been improved by single-point corrections using density functional theory (DFT) methods. Free energy surfaces have been calculated in the framework of the hydride transfer hypothesis. This mechanism involves the transfer of the lactate hydroxyl proton to H373 while the substrate $\alpha \mathrm{H}$ atom is transferred as a hydride to the flavin mononucleotide (FMN) prosthetic group anchored in the active site. Four different systems have been modeled: wild-type enzyme considering R289 in a distal or a proximal conformation observed in crystal structures and the D282N and Y254L variants (with R289 in a distal position). Simulation results highlight the influence of the environment on the catalytic mechanism by describing a step-wise process in the WT enzyme with R289 in a distal position and a concerted mechanism for the other systems. In the step-wise mechanism, the hydride transfer to flavin can occur only after a proton transfer from substrate to H373. Modifications of the electrostatic field around L-lactate or H373 disfavor the highly charged complex resulting from this proton transfer. Simulations of the Y254L variant also reveal some effect of steric changes.

\section{Introduction}

Flavocytochrome $b_{2}$ (L-lactate cytochrome c oxidoreductase, Fcb2) from the mitochondrial intermembrane space of Saccharomyces cerevisiae is a homotetrameric enzyme that catalyses the oxidation of L-lactate to pyruvate. ${ }^{1}$ Each of the four subunits contains a Flavin MonoNucleotide (FMN) prosthetic group and a $b$-type heme localized in two different domains. The first step of the catalytic cycle consists in the oxidation of L-lactate by the FMN prosthetic group. FMN is later reoxidized by transferring its electrons one by one to the ferric heme. The final electron acceptor is cytochrome $c{ }^{1}$ 
This flavoenzyme has been widely studied as a prototype of L- $\alpha$-hydroxy acid-oxidizing enzymes, particularly for its mechanism of lactate oxidation. ${ }^{2}$ Because of great structural similarities and numerous conserved residues in the active site, members of this flavoenzyme family are supposed to share the same chemical mechanism of $\alpha$-hydroxy acids conversion to the corresponding $\alpha$-keto acid by the transfer of two protons and two electrons. A histidine, H373 in Fcb2, is the active site base.

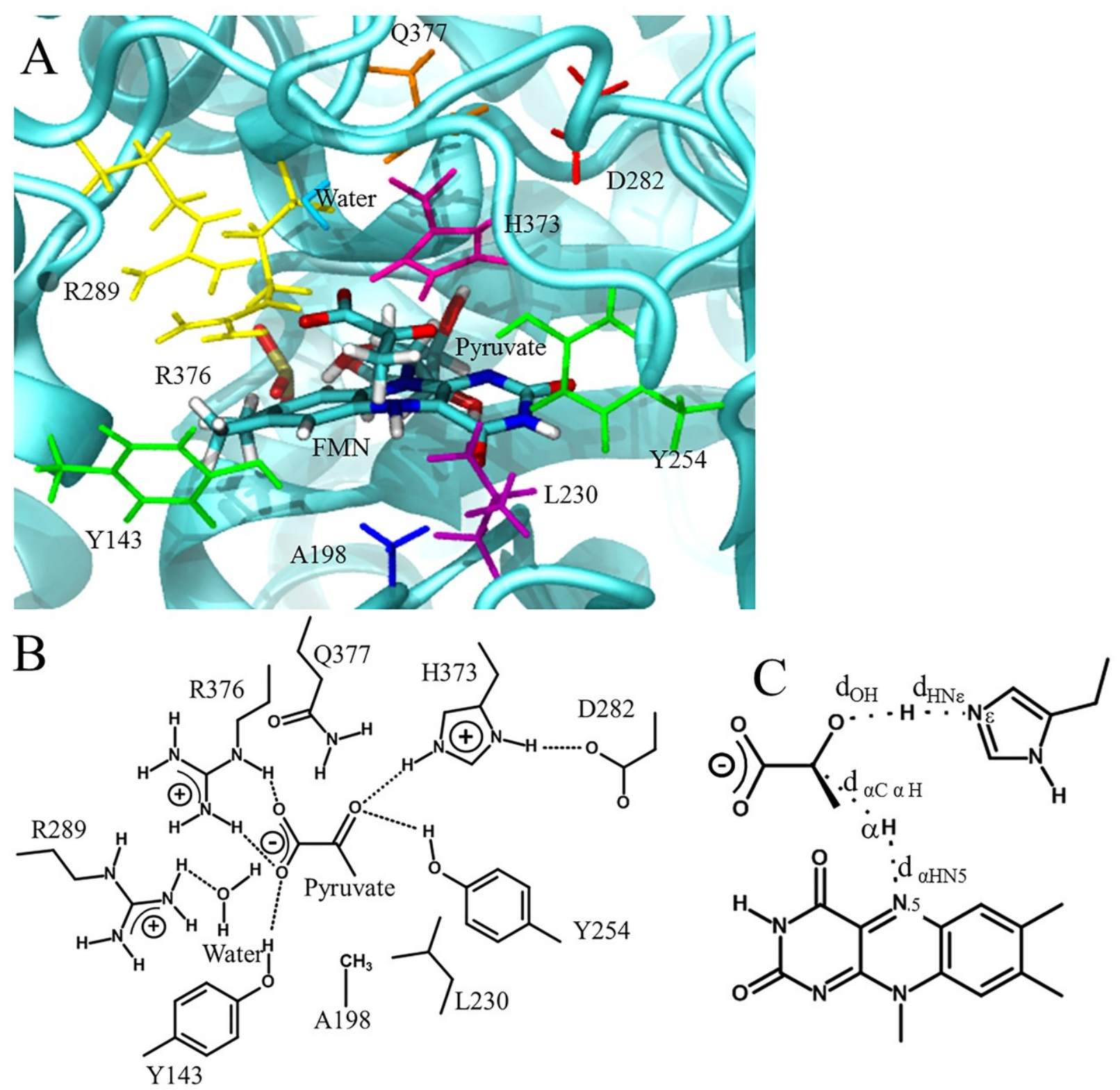

Figure 1: (A) 3D and (B) schematic representation of pyruvate in the Fcb2 active site pocket with $\mathrm{R289}$ in distal conformation from crystal structure $1 \mathrm{KBI}^{3}$ Dashed lines in figure (B) represent possible hydrogen bonds. (C) name of the main atoms involved in the chemical 
process in the active site and scheme of the two reaction coordinates associated with proton transfer (antisymmetrical combination of $\mathrm{O}-\mathrm{H}$ and $\mathrm{O}-\mathrm{H373} \mathrm{N} \varepsilon$ distances) and hydride transfer (antisymmetrical combination of $\alpha \mathrm{C}-\alpha \mathrm{H}$ and $\alpha \mathrm{H}-\mathrm{FMN} \mathrm{N5}$ distances). 3D visualization are treated by VMD. ${ }^{4}$

Several Fcb2 crystal structures ${ }^{3,5}$ show pyruvate, the reaction product, bound in the active site on the flavin si-side, and its interactions with a number of residues. These are displayed in Figure 1. H373 interacts both with the D282 carboxylate and with the pyruvate keto group, suggesting it is doubly protonated. A tyrosine residue, Y143, interacts with one of the pyruvate carboxylate oxygen atoms, while the hydroxyl of another tyrosine, Y254, is hydrogen bonded to the pyruvate keto group in the WT crystal structures. An arginine residue, R376, interacts with the pyruvate carboxylate oxygens. R289 requires particular attention as it has been found in two possible conformations as depicted in Figure 2. In a few crystal structures containing pyruvate, ${ }^{3,5}$ R289 is found in a so-called distal orientation and interacts both with D292 and a water molecule. In other crystal structures, the water molecule disappears while the R289 guanidino group points toward the ligand, forming an electrostatic interaction with the carboxylate. ${ }^{3,6}$ In the crystal structure of the R289K variant in complex with sulfite, the water molecule has been displaced by the lysine side chain, which adopts more or less the orientation of R289 in the proximal position, and interacts with flavin-bound sulfite. ${ }^{7}$ The homologue of R289 is in the proximal position in most structures of family members.
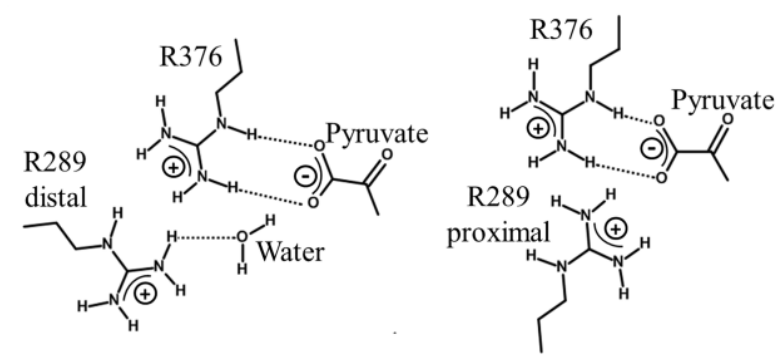

Figure 2: Fcb2 active site arginines around pyruvate. $\mathrm{R} 289$ is represented in its distal orientation (left) or proximal orientation (right). Dashed lines represent possible hydrogen bonds.

Several residues contribute to FMN stabilization in the enzyme pocket (Figure S1 in SI). K349 can particularly influence the enzymatic efficiency by interacting with the ribityl 2'-OH and FMN N1, stabilizing then its negative charge in the reduced enzyme. ${ }^{8}$ In addition, Fcb2 crystal 
structures present mobility of loop 4 of the triosephosphate isomerase (TIM) barrel, at the Cterminal end of the barrel where FMN is bound (Figure S2). Several residues in that proteasesensitive loop are invisible in all structures. ${ }^{3,5-7}$ There is evidence that residues of this loop interact with the active site during catalysis, since the mere proteolysis of one specific peptide bond in the loop induces alterations of the kinetic parameters. ${ }^{9}$

Despite the amount of experimental data collected over the past decades, two mechanistic hypotheses (see Figure 3) remain in debate. ${ }^{2,10-18}$ The first one, so-called "carbanion mechanism", implies the abstraction of the $\alpha$-proton $(\alpha \mathrm{H})$ of L-lactate by the active-site histidine with formation of a carbanion intermediate. The electrons are then transferred to FMN. The other mechanism, known as "hydride transfer mechanism", involves the abstraction of the lactate hydroxyl proton by the histidine and $\alpha$-hydrogen transfer as a hydride to the FMN N5 atom.

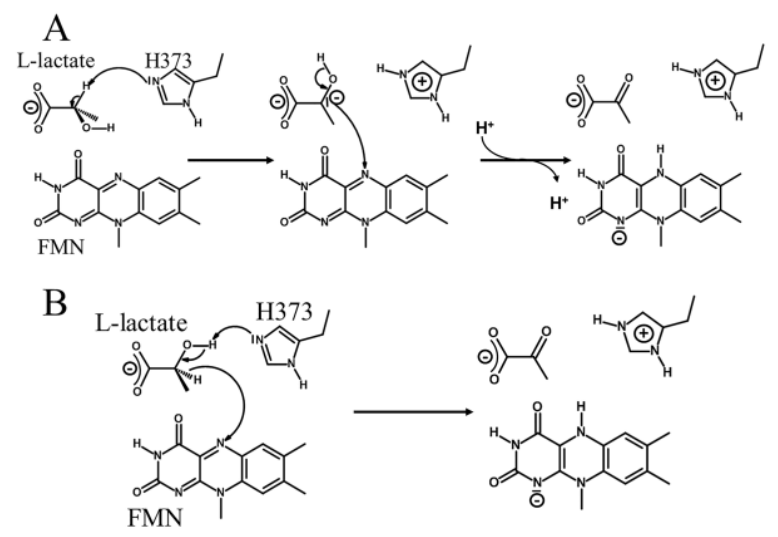

Figure 3: Proposed mechanisms for oxidation of L-lactate in Fcb2: A) carbanion mechanism in which the $\alpha \mathrm{H}$ is abstracted by $\mathrm{H373}$ while the carbanion intermediate gives its electrons to FMN; and B) hydride transfer mechanism in which the hydroxyl proton is abstracted by $\mathrm{H373}$ while $\alpha \mathrm{H}$ is transferred to FMN as a hydride.

Site-directed mutagenesis of some active site residues helped to understand their role in stabilization of the Michaelis complex or the transition state (TS). Studies of Y254F and Y254L variants ${ }^{17,19,20}$ underlined the role of Y254 in TS stabilization while these mutations appeared to have little influence on the Michaelis complex formation. Furthermore, mutation of D282 to asparagine showed that this residue has a key role by stabilizing the H373 imidazolium ion and increasing the $\mathrm{H} 373 \mathrm{pKa}$ in the reduced enzyme. ${ }^{21}$ Finally, the specificity of Fcb2 toward Llactate can be modulated by mutation of two aliphatic residues L230 and A198. ${ }^{22-24}$ Modeling studies suggested that they sterically interfere with a mandelate binding mode for a carbanion 
mechanism and not for a hydride transfer. While the WT enzyme can hardly catalyse Lmandelate oxidation, ${ }^{17}$ individual mutations at these positions as well as the double mutation substantially increased the activity for mandelate, while decreasing that for lactate. The double variant was somewhat more efficient with mandelate than with lactate. ${ }^{23}$

Primary deuterium isotope effects show that the rate-limiting step consists in the transfer of the L-lactate $\alpha$-hydrogen. ${ }^{25,26}$ Solvent kinetic isotope effects were also experimentally determined. ${ }^{26}$ The absence of solvent kinetic isotope effect in the WT enzyme was interpreted as compatible with a carbanion mechanism or a non-concerted hydride transfer. In the presence of both $\left[2-{ }^{3} \mathrm{H}\right]$ lactate and fluoropyruvate under transhydrogenation conditions, Rao et al ${ }^{13}$ determined a $\mathrm{pKa}$ of 9.1 for the $\alpha \mathrm{H}$ acceptor in the reduced enzyme. In addition, this acceptor has its $\mathrm{pKa}$ reduced by $1.4 \mathrm{pH}$ unit in the $\mathrm{D} 282 \mathrm{~N}$ variant. ${ }^{21}$ These results are more consistent with the $\alpha \mathrm{H}$ acceptor being $\mathrm{H} 373$ rather than FMN N5, since in the free reduced flavin the N5 pKa is $\geq 20 .^{27}$

Computational simulations could provide a molecular description of L-lactate in the Fcb2 active site and should contribute to characterize the oxidation mechanism at a microscopic level. A first computational study was published by Tabacchi et al. ${ }^{28}$ based on Density Functional Theory Car-Parrinello Molecular Dynamics simulations of an active site model (169 atoms). The carbanion hypothesis was not tested as no optimized Michaelis complex structure was found to be compatible with this mechanism. Moreover, only the distal orientation of R289 was fully studied as the proximal orientation presented a higher potential energy $(11.2 \mathrm{kcal} / \mathrm{mol})$ at the reactant state. Comparison between different models showed the contribution to the stabilization of the reduced flavin of an interaction between the FMN ribityl 2'-OH and K349 as well as that of an invariant water molecule bridge between D282 and S371. Using the "bluemoon ensemble" approach $^{29}$ combined with First Principle Molecular Dynamics, $^{30}$ the authors reported a onedimensional free energy profile along a geometrical reaction coordinate associated to $\alpha \mathrm{H}$ transfer to FMN. Proton transfer to H373 was not constrained. The results suggested that the reaction occurs via a concerted but asynchronous mechanism where the $\alpha$-hydroxyl proton transfer to H373 precedes the hydride transfer to FMN assisted by the isoalloxazine $\mathrm{C} 4 \alpha-\mathrm{C} 10 \alpha$ 'electronpaved path, ${ }^{31}$ The reported activation free energy value was $12.1 \mathrm{kcal} / \mathrm{mol}$ which was compatible with the estimated experimental value of 13.5-13.6 $\mathrm{kcal} / \mathrm{mol}$. Nevertheless, despite the quantitatively good agreement, the employed QM model suffers from its limited size thus 
ignoring the effect of residues or solvent water molecules that could influence the reaction and the active site conformation. For instance, some interactions could counterbalance the loss of the hydrogen bond between R289 and D292 when R289 is turned from distal to proximal conformation.

We herein present an extensive QM/MM study of the putative hydride transfer mechanism of L-lactate oxidation to pyruvate catalysed by Fcb2 in order to get an insight on the origin of catalysis in this enzyme considering the whole Fcb2 flavodomain in water. Free energy surfaces were computed as two-dimensional potential of mean force along selected geometrical coordinates associated to each proton and hydride transfer using molecular dynamics (MD) simulations. Two different structures of the protein, with distal and proximal conformations of R289, have been used as starting point of our simulations. The computational protocol was also applied to variants such as Y254L and D282N, which have been studied experimentally, ${ }^{17,21,26}$ in order to understand the role of specific active site residues. Further studies of the carbanion mechanism could be done in the future to support our proposals based on the exploration of the hydride transfer mechanism.

\section{Computational methods}

Our simulations use the flavin domain B chain of the 1KBI (pdb code) structure of Fcb2, which contains pyruvate in the active site. The piece of loop 4 peptide chain between residues 301 and 308, which is invisible in this structure was reconstructed and the residues conformation optimized by minimization and molecular dynamics (MD) simulations. Hydrogen atoms have been added using fDYNAMO ${ }^{32}$ package according to $\mathrm{pKa}$ values of titratable residues from PROPKA program $3.0 .^{33,34}$ To neutralize the system, six sodium counter ions were placed into an optimal electrostatic position around the enzyme. The protein and the ions have then been solvated in a $130 \times 130 \times 130 \AA$ water box. All water molecules with oxygen atom lying less than $2.8 \AA$ away from any heavy atoms have been deleted. The set of atoms treated quantum mechanically or QM region, described by the AM1 semiempirical Hamiltonian, ${ }^{35}$ comprises the entire flavin, the substrate lactate (replacing the pyruvate of the structure) and the H373 sidechain. A hydrogen quantum link atom was inserted in the $\mathrm{C} \alpha-\mathrm{C} \beta$ bond of $\mathrm{H} 373$, where the QM/MM boundary intersected covalent bonds. ${ }^{36}$ Keeping in mind that no other fragment of the protein active site is going to be involved in forming or breaking of covalent bonds, or in important charge transfer, just this link atom has been included. The rest of the system was 
simulated employing the OPLS-AA ${ }^{37}$ and TIP3P ${ }^{38}$ force fields for the protein and water molecules, respectively. The full system was then formed by $73 \mathrm{QM}$ atoms, 6500 protein atoms, 6 sodium counter ions and 55719 water molecules. Cut-off for non-bonded interactions were applied using a switching-force scheme with a range of radius from 12 to $13.5 \AA$. All the atoms farther than $30 \AA$ from any of the atoms of the QM part have been fixed in all the calculations. In order to study the conformation of the protein corresponding to R289 in a proximal orientation, one crystallographic water molecule inside the active side was removed and the Arg289 conformation was manually modified. The system was finally equilibrated at $300 \mathrm{~K}$ during 500 ps of QM/MM MD simulations using Langevin-Verlet integrator in NVT ensemble and a time step of 0.5 fs.

The Potential Energy Surfaces (PES) have been obtained by optimizing geometries along two reaction coordinates: the antisymmetric combination of the distances defining the $\mathrm{O}-\mathrm{H}$ bondbreaking and the $\mathrm{H}-\mathrm{H} 373 \mathrm{~N} \varepsilon$ bond-forming, (Proton RC), and those defining the $\alpha \mathrm{C}-\alpha \mathrm{H}$ bond breaking and the $\alpha \mathrm{H}-\mathrm{FMN} \mathrm{N} 5$ bond forming (Hydride RC):

Proton $R C=d_{O H}-d_{H N \varepsilon}$

Hydride $R C=d_{\alpha C \alpha H}-d_{H \alpha N 5}$

These distances were constrained during optimization using a harmonic force of 10000 $\mathrm{kJ} / \mathrm{mol} / \AA^{2}$. Free energy surfaces (FES), computed in terms of two dimensional Potential of Mean Force (2D-PMF), were calculated from PES geometries, using an umbrella sampling approach ${ }^{39}$ with a force constant of $2500 \mathrm{~kJ} / \mathrm{mol} / \AA^{2}$ on each reaction coordinate. Each window of simulation consisted in 10 ps of equilibration followed by 20 ps of production with a time step of $0.5 \mathrm{fs}$. The weighted histogram analysis method (WHAM) ${ }^{40}$ was used to get the full probability distribution function. Because of the large number of structures that must be evaluated during free energy calculations, QM/MM calculations are usually restricted to the use of semiempirical Hamiltonians. In order to correct the low-level AM1 energy function used in the 2D-PMFs, an interpolated correction scheme developed in our laboratory was applied. ${ }^{41,42}$ In this correction scheme, based on a method proposed by Truhlar and co-workers for dynamical calculations of gas phase chemical reactions, ${ }^{43-45}$ a spline under tension ${ }^{46,47}$ is used to interpolate this correction term at any value of the distinguished reaction coordinates, $\zeta$, selected to generate the free energy surfaces. The new energy function employed in the simulations is defined as: 


$$
E=E_{L L / M M}+S\left[\Delta E_{L L}^{H L}(\zeta)\right]
$$

where $S$ denotes a spline function, whose argument $\Delta \mathrm{E}_{\mathrm{LL}}^{\mathrm{HL}}(\zeta)$ is a correction term taken as the difference between single-point calculations of the QM subsystem using a high-level (HL) method, and the low-level (LL) one. The correction term is expressed as a function of the distinguished reaction coordinate $\zeta$ (RC). When corrections were done on 2D-PMF, the correction term is then expressed as a function of the two coordinates $\zeta_{1}, \zeta_{2}$ that were employed to generate the lower level 2D-PMF. The HL calculations were carried out by means of the M06-2X functional ${ }^{48}$ with the $6-31+\mathrm{G}(\mathrm{d}, \mathrm{p})$ basis set ${ }^{49,50}$ using Gaussian 09 program. ${ }^{51}$ This protocol has been demonstrated to provide good results in different systems. ${ }^{52,53}$

Once the corrected 2D-PMF were obtained, 500 ps of MD were performed for each identified stationary point on the FES. Distances and electrostatic interactions between the active site and closed residues were collected along the simulations. For MD on the TSs, a harmonic constraint with a constant force of $2500 \mathrm{~kJ} / \mathrm{mol} / \AA^{2}$ was applied to each reaction coordinate.

\section{RESULTS AND DISCUSSION}

Wild type enzyme (WT). First, the orientation of R289 and the presence of a water molecule in the active site must be clearly defined since this deeply modifies the interactions around the substrate carboxylate. In the distal R289 case, the carboxylate group is stabilized by electrostatic interaction and two hydrogen bonds with R376, one hydrogen bond with Y143 and a polar interaction with a water molecule perpendicular to the carboxylate plane. In the R289 proximal case, the positive R289 guanido group faces the carboxylate, adding a strong charge interaction around it. In this latter conformation, the negative charge of L-lactate is supposed to be better stabilized than in the R289 distal position. This stabilization should lead to a decrease of the electron density around the $\alpha$-carbon, which would become more electrophilic. Consequently, the reactivity of L-lactate in the cavity should strongly depend on the conformation of R289. The most efficient conformation will also serve as reference for the simulations of the variant proteins.

Looking at the crystal structures, it appears that both the R376 backbone and the Q377 side chain amide can form hydrogen bonds with the R289 guanido group in the proximal 
orientation while R289 is stabilized in the distal orientation by an interaction with D292. In their model, Tabacchi et $a l^{28}$ represent D292 but omit Q377 and the R376 backbone. They found the active site is less stable by a tenth of $\mathrm{kcal} / \mathrm{mol}$ with R289 in a proximal position. Our QM/MM scheme allows the treatment of all the interactions around R289 in both orientations at a classical level so that the equilibrium between the two orientations can differ from the first computational study.

The first AM1/MM PES we obtained concerns the enzyme with R289 in a distal position (Figure S3 in Supporting Information). As explained in the Computational Methods section, geometries taken from this surface were used as seed to generate the corresponding 2D-PMF.
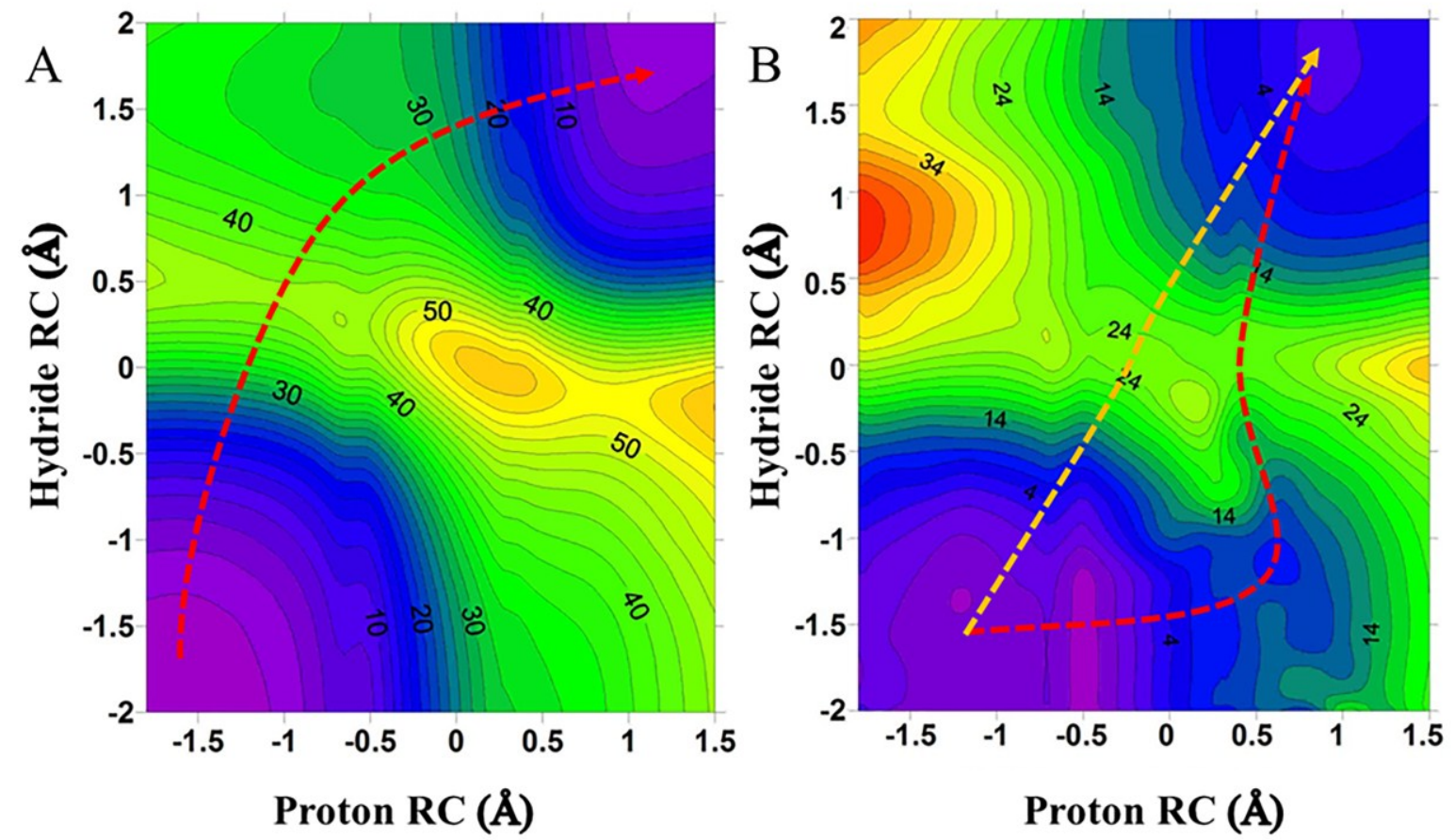

Figure 4: Free energy surfaces of a hydride transfer mechanism in Fcb2 for a distal position of R289, computed as 2D-PMF at AM1/MM (A) and M06-2X:AM1/MM (B) level at $300 \mathrm{~K}$. Isoenergetic lines are drawn for each $2 \mathrm{kcal} / \mathrm{mol}$, from purple minima to red maxima. 

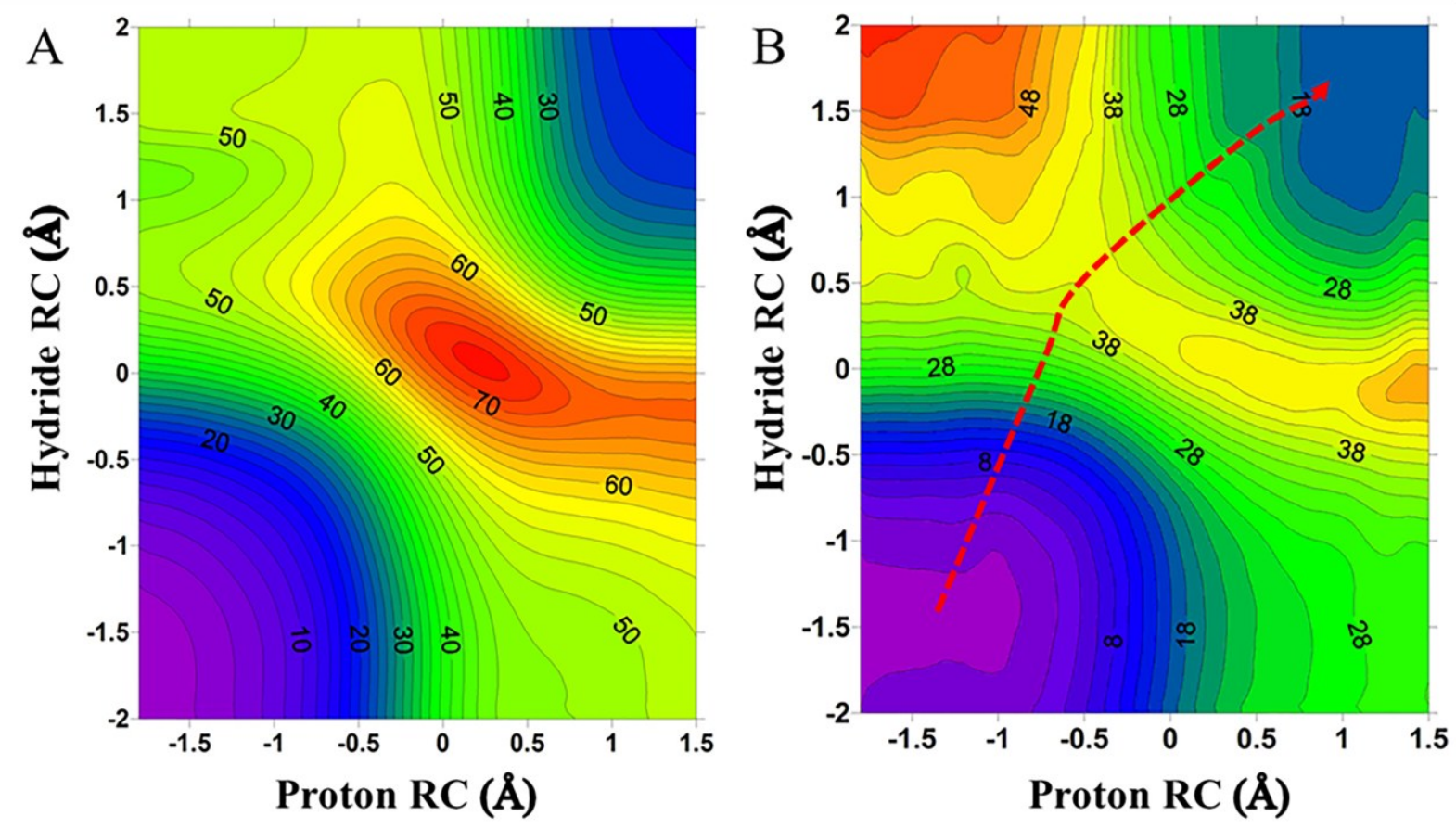

Figure 5: Free energy surfaces of a hydride transfer mechanism in Fcb2 for a proximal position of R289, computed as 2D-PMF at AM1/MM (A) and M06-2X:AM1/MM (B) level at $300 \mathrm{~K}$. Isoenergetic lines are drawn for each $2 \mathrm{kcal} / \mathrm{mol}$, from purple minima to red maxima.

The 2D-PMF obtained for the oxidation of L-lactate to pyruvate catalysed by WT Fcb2 are presented in Figure 4 and Figure 5 for the conformation of Fcb2 with distal and proximal position of R289, respectively. As observed in Figure 4A, the FES obtained at AM1/MM level describes a concerted but asynchronous mechanism. When the FES was computed at M062X:AM1/MM level, see Figure 4B, the reaction is still described by a concerted mechanism, but the position of the TS is shifted to a more synchronous reaction mechanism. Moreover, now an additional competitive reaction path can be traced on the surface describing a step-wise mechanism where the hydride transfer takes place only when the proton is close to the histidine $\mathrm{N} \varepsilon$ atom. This first step costs around 12-13 kcal/mol (maximum localized at values of proton RC of $0.5 \AA$ and hydride RC of $-1.3 \AA$ ) and it is reversible with almost no energy expense. The proton can quite easily move between its two acceptors (L-lactate and H373), indicating that the system oscillates between reactants and a shallow minimum. In a second step, the products are reached from this minimum. This second step is the limiting step of the reaction with a maximum of free energy localized at values of proton RC of $0.5 \AA$ and hydride RC of $0.1 \AA$ and 29.5 
$\mathrm{kcal} / \mathrm{mol}$ higher than the reactants state (Table 1). A similar change in the description of a reaction mechanism from results obtained at $\mathrm{AM} 1 / \mathrm{MM}$ to a higher level treatment was already observed in our laboratory when exploring the reduction of pyruvate into lactate catalysed by a nicotinamide-dependent lactate dehydrogenase, $\mathrm{LDH}$, on $\mathrm{PESs}^{54}$ and on FESs. ${ }^{55}$ The final product with pyruvate is less stable than reactants, suggesting that it is less stabilized in the active site. These results are coherent with those previously obtained by Tabacchi et $a l^{28}$ and the fact that no solvent isotope effect was encountered in this system experimentally. ${ }^{26}$ For completeness, in a recent study, Cao et al calculated 2D-PES of L-lactate oxidation in Long Chain L- $\alpha$-Hydroxy Acid Oxydase (LCHAO), a member of the L- $\alpha$-hydroxy acid-oxidizing enzymes family. ${ }^{56}$ They used DFT/MM optimizations along two geometrical reaction coordinates corresponding to each transfer. Their results describe an asynchronous mechanism in WT enzyme, similar to our red one (see Figure 4B), starting with the proton transfer and reaching a shallow intermediate from which the hydride can be transferred.

The other pathway (yellow line in Figure 4B) consists in simultaneous proton and hydride transfers and is associated with a free energy of activation of $29.8 \mathrm{kcal} / \mathrm{mol}$. Thus the global free energies of activation are equivalent in the two pathways, but the asynchronous one seems to be the most favourable one. Indeed, the system can easily transfer the proton from substrate to catalytic base and this new conformation, slightly stabilized, can serve as a starting point for hydride transfer from lactate to flavin. A negative charge on FMN would be stabilized thanks to the positive charge of protonated H373 stacked upon the isoalloxazine ring. ${ }^{57}$ In the following, the TS associated to WT system with R289 in a distal position refers to the TS of the hydride transfer in the step-wise mechanism.

$k_{\text {cat }} \exp \left(\mathrm{s}^{-1}\right) \quad \Delta \mathrm{A}^{\ddagger}$ calc $(\mathrm{kcal} / \mathrm{mol})$

\begin{tabular}{ccc}
\hline WT R289 distal & 29.5 \\
WT R289 proximal & & 42.2 \\
D282N & $3.9 \pm 0.1^{21}$ & 32.9 \\
Y254L & $0.51 \pm 0.02^{17}$ & 36.7
\end{tabular}

Table 1: experimental steady-state kinetic parameters, theoretical activation free energies for L-lactate oxidation in WT, D282N and Y254L variant. For experimental data, 
ferricyanide is used as electron acceptor (concentration 1 or $2 \mathrm{mM}$ ) in a standard buffer $\left(0.1 \mathrm{M} \mathrm{Na}^{+} / \mathrm{K}^{+}\right.$phosphate, $1 \mathrm{mM}$ EDTA, $\mathrm{pH}$ 7) at $30^{\circ} \mathrm{C}$. The WT rate constant includes the step o flavin reoxidation.

\begin{tabular}{|c|c|c|c|c|c|c|c|c|c|c|c|c|}
\hline & \multicolumn{3}{|c|}{ WT R289 distal } & \multicolumn{3}{|c|}{$\begin{array}{l}\text { WT R289 } \\
\text { Proximal }\end{array}$} & \multicolumn{3}{|c|}{ D282N } & \multicolumn{3}{|c|}{ Y254L } \\
\hline & $\mathrm{R}$ & $\mathrm{TS}$ & $\bar{P}$ & $\overline{\mathrm{R}}$ & $\mathrm{TS}$ & $\bar{P}$ & $\bar{R}$ & $\mathrm{TS}$ & $\bar{P}$ & $\overline{\mathrm{R}}$ & $\mathrm{TS}$ & $\bar{P}$ \\
\hline $\mathbf{H}_{\text {LAC }}-\mathbf{O}_{\text {LAC }}$ & 0.97 & 1.56 & 2.41 & 0.97 & 1.08 & 4.52 & 0.97 & 1.01 & 2.43 & 0.96 & 1.04 & 3.3 \\
\hline $\mathrm{H}_{\mathrm{LAC}}-\mathrm{N} \varepsilon \mathrm{H} 373$ & 3.23 & 1.12 & 1.01 & 5.45 & 1.6 & 1.01 & 3.77 & 1.79 & 1.02 & 4.35 & 1.76 & 1 \\
\hline$\alpha H-\alpha C$ & 1.13 & 1.44 & 3.89 & 1.13 & 1.61 & 3.84 & 1.13 & 1.45 & 4.16 & 1.13 & 1.62 & 4.12 \\
\hline H $\alpha-N 5 F M N$ & 3.37 & 1.26 & 1.01 & 4.23 & 1.13 & 1.01 & 3.81 & 1.24 & 1.01 & 4.52 & 1.14 & 1.01 \\
\hline O1-NعR376 & 2.9 & 2.94 & 2.94 & 3.11 & 3.07 & 3.85 & 2.89 & 2.91 & 2.95 & 2.87 & 2.93 & 2.81 \\
\hline O2-NH2R376 & 2.89 & 2.86 & 2.93 & 2.85 & 2.96 & 2.96 & 3.01 & 2.91 & 2.94 & 2.9 & 2.94 & 3.01 \\
\hline O1-NH2R289 & 4.77 & 6.06 & 4.41 & 2.88 & 2.91 & 2.95 & 3.78 & 4.18 & 4.52 & 4.39 & 6.31 & 6.24 \\
\hline O2-NH1R289 & 5.66 & 6.21 & 4.76 & 2.99 & 2.98 & 2.93 & 5.03 & 5.2 & 4.89 & 5.06 & 6.39 & 6.73 \\
\hline O2-OY143 & 2.95 & 3.11 & 4.42 & 3.12 & 2.97 & 4.33 & 3.29 & 2.89 & 3.93 & 3.34 & 3.48 & 3.7 \\
\hline O1-WAT491 & 2.91 & 3.15 & 3 & & - & & 2.85 & 2.93 & 3.07 & 2.9 & 3.73 & 3.33 \\
\hline O3-OY254 & 3.04 & 2.82 & 3.08 & 4.32 & 3 & 3.58 & 3.84 & 2.9 & 3.15 & 7.74 & 6.65 & 6.73 \\
\hline N反H373-OD289 & 3.44 & 3.18 & 2.93 & 3.33 & 3.15 & 3.24 & 3.58 & 3.41 & 3.68 & 3.71 & 3.86 & 3.3 \\
\hline
\end{tabular}

Table 2: Average intra and intermolecular distances in the active site from MDs simulations at reactants (R), transition state (TS) or products (P) for WT, D282N and Y254L Fcb2. For WT enzyme with R289 in a distal position, TS refers to the transition state of the hydride transfer step of the step-wise mechanism. Distances are provided in ångströms. More detailed information is given in Table $\mathrm{S1}$. 


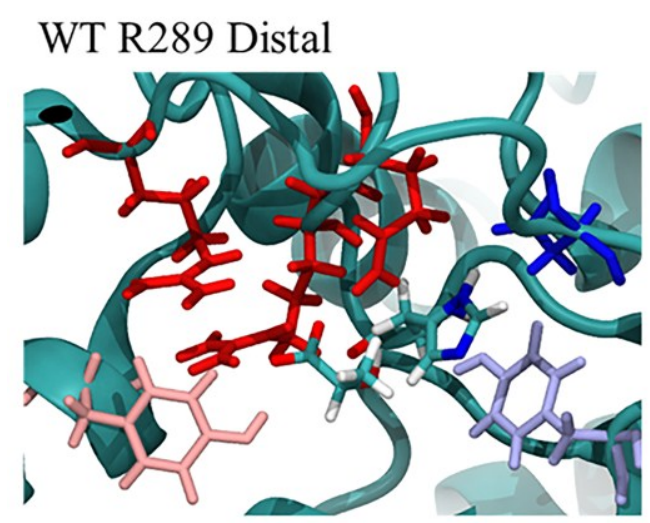

WT R289 Proximal
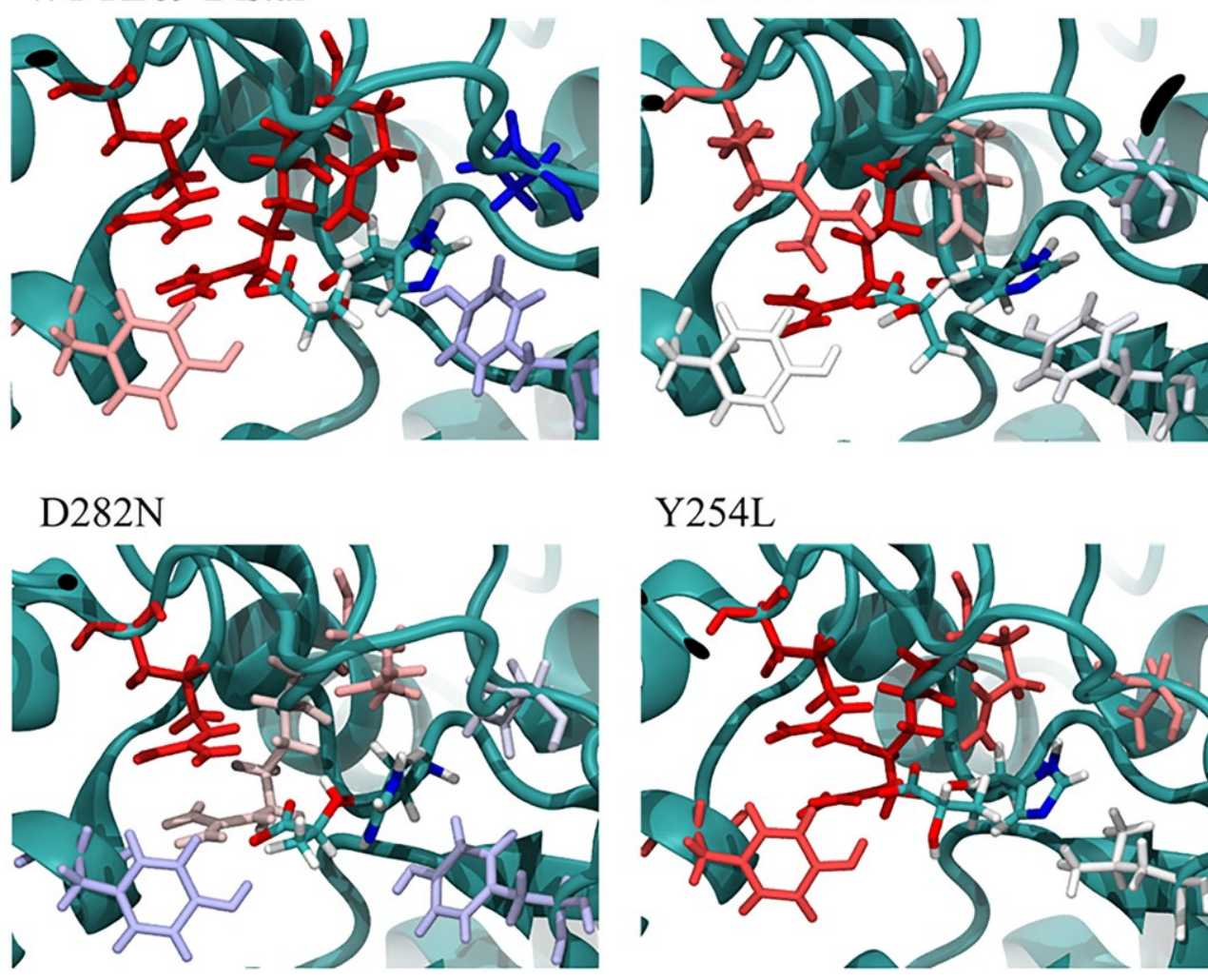

$\Delta \mathrm{E}_{\text {elec }}(\mathrm{R}-\mathrm{TS})$

Y254L

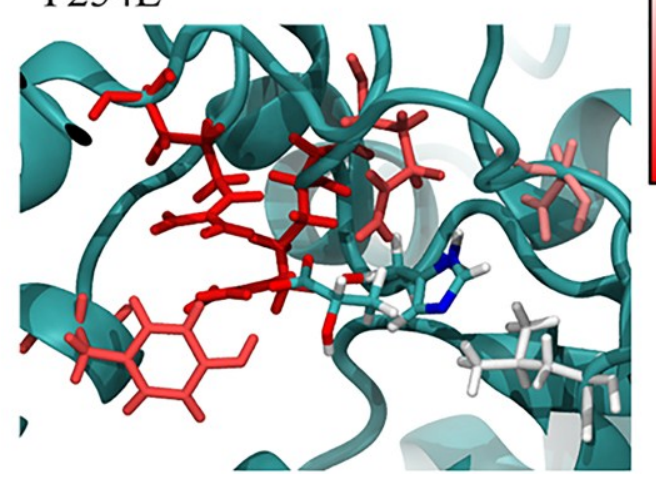

$0 \mathrm{kcal} / \mathrm{mol}$

$-10 \mathrm{kcal} / \mathrm{mol}$

Figure 6: Fcb2 variants active site residues coloured as a function of the QM part/classical residues average electrostatic interaction energy difference between reactants and TS MD simulations. Red colour corresponds to a decreasing interaction from reactants to TS, blue colour to an increasing attractive interaction and white colour to a constant interaction. Structures are picked from MD simulation of reactants in each Fcb2 variant.

When R289 is in the proximal conformation (see Figure 5A), no transition state could be clearly defined in the AM1/MM FES for a concerted mechanism. At M06-2X:AM1/MM level, a concerted path can be traced on the surface presented in Figure 5B. The maximum of this concerted path appears at values of proton $\mathrm{RC}$ of $-0.4 \AA$ and hydride $\mathrm{RC}$ of $0.5 \AA$ (see Figure 5B). The free energy surface presents a free energy of activation of $42.2 \mathrm{kcal} / \mathrm{mol}$, about 13 $\mathrm{kcal} / \mathrm{mol}$ higher than the step-wise or concerted path free energies of activation obtained when $\mathrm{R} 289$ is in a distal orientation. This conformation consequently appears to be less favourable to the reaction process. Average distances and interactions between the quantum part and different residues of the active site at reactants, TS and products (Table 2, Figure 6, Table S1 and Table S2), underline how the R289 conformation affects reactivity. When this residue is in a proximal 
position, the substrate carboxylate is attracted equally by the two arginines and the L-lactate alcohol moiety remains too far from Y254 to interact with it (about $4.3 \AA$ in the reactant state). In this position, we observe a decrease of steric hindrance around lactate allowing rotation around the $\mathrm{C}-\alpha \mathrm{C}$ bond. Besides, as H373 and Y254 do not interact with lactate, a hydrogen bond can be formed between the histidine $\mathrm{N} \varepsilon$ and tyrosine hydroxyl. Such a reactant conformation disfavours a proton transfer between the substrate and the catalytic base, whereas $\alpha \mathrm{H}$ is also most of the time quite far from N5 FMN; hence the cost is more expensive for reaching an efficient conformation for two-protons/two-electrons transfers. On the contrary, this conformation would be better suited for a carbanion mechanism, with a more electrophilic $\alpha \mathrm{C}$, an easier rotation of the substrate and a lack of stabilization of the negatively charged alcohol after proton transfer.

In Figure 6, the active site residues are coloured as a function of the difference between reactants and TS of the electrostatic interactions between them and the QM part. For the two arginines, this difference is not deeply affected by the orientation of R289 and corresponds to a decrease from reactants to TS. For R289 in the distal position, H373 is positively charged at the TS while H373 is neutral when R289 is in the proximal position. Consequently, for the R289 distal orientation, the difference of interaction for D282 is bigger than for the R289 proximal position. Y143 and Y254 interactions with the QM part do not much differ from reactants to TS, particularly when R289 is in the proximal orientation.

When R289 is in the distal position, the presence of the water molecule prevents a direct interaction between R289 and lactate and thus a displacement of the substrate towards the two arginines R376 and R289. Maintaining R289 in a distal conformation allows proton transfer to take place before hydride motion. It guarantees a more favourable catalytic interaction between the substrate and H373, Y254 and FMN. Consequently, all the following calculations were performed with R289 in a distal position and in the presence of the crystallographic water molecule.

\section{D282N variant}

D282 is expected to stabilize the imidazolium ion in transition and product states by electrostatic interactions and hydrogen bonding with the $\mathrm{H} \delta$ of $\mathrm{H} 373 .{ }^{21}$ Its mutation to asparagine prevents the electrostatic interaction and decreases the $k_{\text {cat }}$ value by a factor of about 70 compared to WT enzyme. Our 2D-PMF for the D282N variant is presented in Figure 7. 


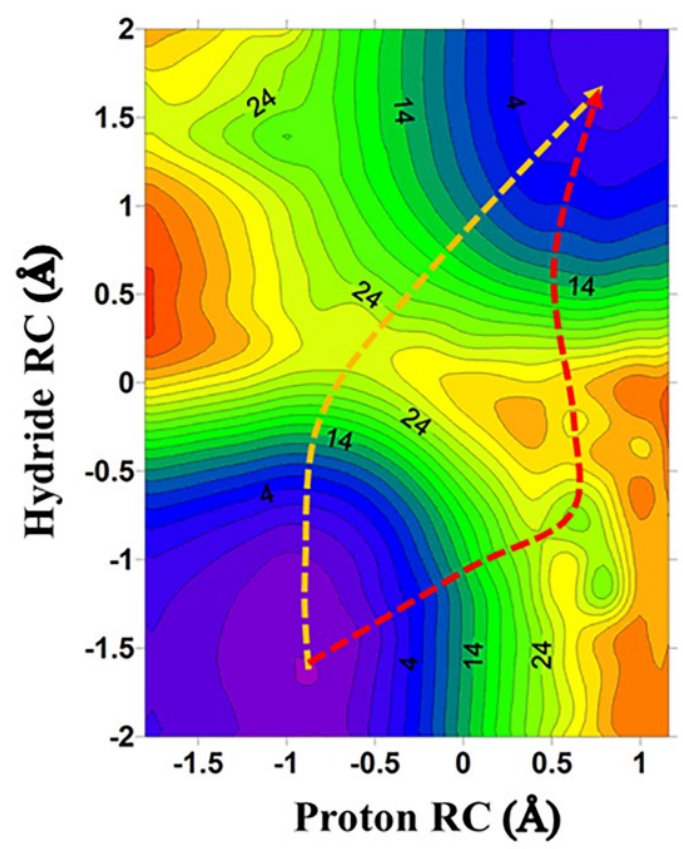

Figure 7: M06-2X:AM1/MM 2D-PMF performed at $300 \mathrm{~K}$ of a hydride transfer mechanism for the Fcb2 D282 variant with $\mathrm{R} 289$ in a distal position. Isoenergetic lines are drawn for each $2 \mathrm{kcal} / \mathrm{mol}$, from purple minima to red maxima.

We meet again two possible pathways, the same as previously described for the WT. However, in this variant, the concerted pathway seems to be the favoured one, with a free energy of activation of $32.9 \mathrm{kcal} / \mathrm{mol}$ (Table 1). Following the step-wise mechanism, the first proton transfer costs about $32 \mathrm{kcal} / \mathrm{mol}$ while we have to add $8 \mathrm{kcal} / \mathrm{mol}$ to transfer the hydride anion in a second step of the reaction (red pathway). The free energy of activation obtained using our model is overestimated compared with the experimental data. Indeed, our results predict a rate constant difference of three orders of magnitude between WT and D282N enzymes instead of the two orders of magnitude that were experimentally measured. We also optimized the TS structure for the different systems at M06-2X/MM level. We found that they are located on the quadratic region of the DFT free energy surfaces (Figure S4), supporting our computational protocol based on spline corrections and giving credence to our results.

In the D282N variant, the asparagine does not electrostatically interact with the quantum part (see Figure 6 and Table S2). H373 must thus present a lower pKa, unable to attract the substrate hydroxyl proton. However, electron transfer from lactate to FMN may aid the proton transfer. As FMN and H373 are $\pi$-stacked, they can interact to stabilize their respective charges. 
We previously saw that the positive histidine can assist isoalloxazine reduction. Here, conversely, once flavin is reduced, or semi-reduced, its negative charge can stabilize the positive histidine. This requires that at least one electron transfer from lactate to flavin before proton transfer to H373. $\alpha \mathrm{H}$ motion from lactate can allow this electron transfer in the concerted pathway.

At the reactant state, distances between proton or hydride donor and acceptor are quite close to those in the WT enzyme one (Table 2). Indeed, the D282N mutation does not modify steric hindrance around the substrate. It only plays a role in the electrostatic stabilization of the product after proton transfer from the lactate hydroxyl to H373 NE. Nevertheless, electrostatics is not the only factor that can play a role on the catalytic oxidation of lactate by Fcb2. Other variants were generated experimentally to modify steric hindrance in the active site: Y254L, L230A, A198G/L230A, etc. with effects on both dissociation and rate constant. ${ }^{17,22-24}$

\section{Y254L variant}

Y254 is proposed to bind to the substrate hydroxyl and to stabilize the TS via a hydrogen bond. The mutation of this residue to phenylalanine, which removes the hydrogen bond without modifying the size of the residue, decreases the $k_{\text {cat }}$ about 40 -fold compared to WT but the reaction remains possible, underlying that the hydrogen bond between Y254 and lactate is not crucial. ${ }^{17,20}$ However, the Y254L mutation leads to a $k_{\text {cat }}$ value 500 -fold lower than in the WT enzyme. In this variant, the steric hindrance due to the residue at position 254 is reduced around the lactate $\alpha \mathrm{C}$ atom. Surprisingly, these mutations of $\mathrm{Y} 254$ do not affect the substrate dissociation constant $\mathrm{K}_{\mathrm{M}}$. Both phenol group and aromatic ring of Y254 appear important to lower TS energy, but they are less crucial in Michaelis complex formation. ${ }^{17}$ 


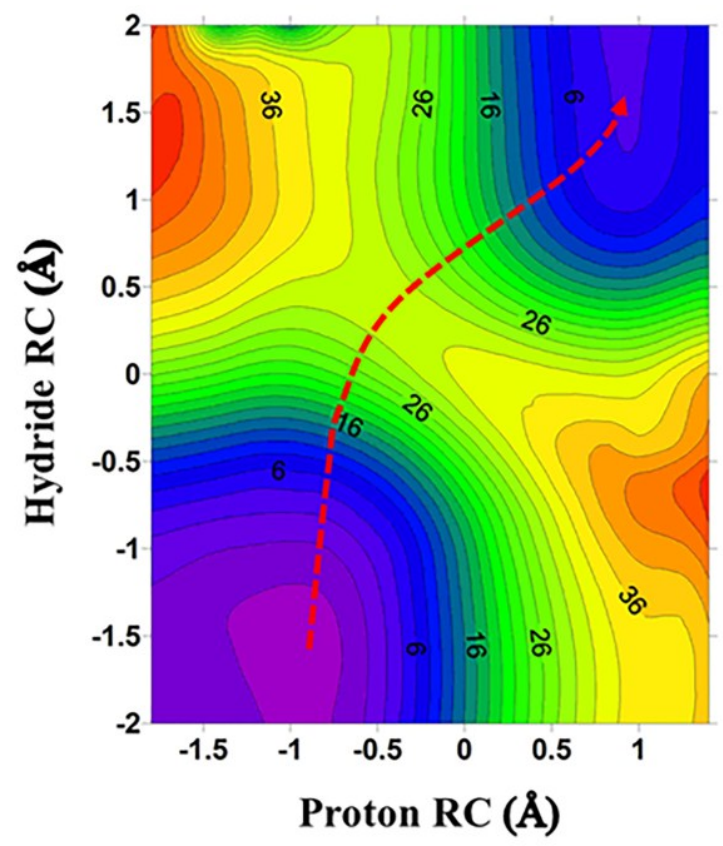

Figure 8: M06-2X:AM1/MM 2D-PMF performed at $300 \mathrm{~K}$ of hydride mechanism in Fcb2 for Y254L variant with R289 in a distal position. Isoenergetic lines are drawn for each $2 \mathrm{kcal} / \mathrm{mol}$, from purple minima to red maxima.

In the 2D-PMF presented in Figure 8, corresponding to L-lactate oxidation by the Y254L variant, only a concerted pathway seems worth considering. The reactants are connected to products through a single TS localized at values of proton RC of $-0.4 \AA$ and hydride $\mathrm{RC}$ of $0.4 \AA$ The proton remains linked to L-lactate and next to H373 (around $1.8 \AA$ at TS) but hydride transfer occurs first, followed by a lower free energy of activation proton transfer. The associated free energy of activation is of $36.7 \mathrm{kcal} / \mathrm{mol}$ (Table 1). This provides a rate constant five order of magnitude lower than the WT ones. Experimentally, the decrease is only about 500 fold. Note finally that even though the computed free energies of activation appear as rather large, they do not rule out the hydride mechanism. In Fcb2, with the hypothesis of a hydride transfer mechanism, our simulations show that both mutations of Y254 and D282 to a nonpolar or neutral residue respectively lead to a modification of the most efficient reaction pathway from step-wise to concerted and thus to a different TS.

With the hydride transfer mechanism hypothesis, Y254 plays a role in the catalytic process by stabilizing the product of the first proton transfer from substrate to H373. In the absence of this stabilization, proton transfer can occur only after hydride departure, as described 
before in the proximal R289 conformation in the WT enzyme. A decrease of steric hindrance around $\alpha \mathrm{C}$ is also observed in the $\mathrm{Y} 254 \mathrm{~L}$ variant. The possible rotation around $\mathrm{C}-\alpha \mathrm{C}$ bond makes a catalytic conformation more difficult to reach. This fact could explain why Y254F variant appears more efficient than the Y254L one. Average distances obtained during MD simulations on the reactant state (Table 2) confirm similarities of substrate binding between the Y254L variant and the WT with R289 in the proximal conformation: absence of interaction between Llactate and proton or hydride acceptors, decrease of the steric hindrance around $\alpha \mathrm{C}$. Besides, the TSs are also similar. Nevertheless, the associated free energies of activation remain different; direct interaction between L-lactate and R289 plays not only on the substrate position in the active site but also on lactate reactivity. Indeed, by making $\alpha \mathrm{C}$ more electrophilic, this interaction contributes to stabilize electronic density around this atom and could disfavour an electron transfer from L-lactate.

The step-wise mechanism appears unlikely in the Y254L variant because of the lack of electrostatic stabilization at the TS and of the difficulty to reach a good conformation for the catalytic process as proton or hydride donor and acceptor can quite easily move and not necessarily facing each other. 2D-PES curve calculated by Cao et al for the Y129F variant of

LCHAO also indicate a concerted mechanism as a minimal energy path. ${ }^{56}$ Besides, if Y254 can contribute to orient the substrate in a proper conformation, its interaction with the QM part remains very low compared to interactions between the two arginines and the QM part, independently of the R289 position. Its mutation to leucine modifies by only $4-5 \mathrm{kcal} / \mathrm{mol}$ the lactate stabilization in the active site, which represents about 2-3\% of the sum of arginines contributions (Table S2). This observation agrees with the small effect of Y254 mutations on dissociation constants.

\section{Role of other residues}

Our results also bring information concerning the influence of other residues of the active site. It has been proposed that Y143 mostly participates to the formation of the Michaelis complex and also facilitates the electron transfer from flavin to heme. ${ }^{58,59}$ In our simulations, Y143 interacts with the substrate carboxylate in the reactant state but the hydrogen bond becomes weaker along the reaction to finally disappear in the products. Consequently, pyruvate is less stabilized by Y143 in the active site than lactate, making the product releases easier. 
In our MD simulations, Q377 residue seems to have a stabilizing interaction with the active site, particularly with H373. In the crystallographic structures, the Q377 side chain amido group oxygen is H-bonded with one of the R289 guanido group nitrogens and the Q377 amido nitrogen atom could interact with the main chain carbonyls of D282 and S285. During our long MDs, the Q377 side chain amido group nitrogen sometimes moves toward H373 and/or the substrate hydroxyl. The associated electrostatic interaction is quite strong at the reactants for all Fcb2 variants and decreases at the TS (Figure 6). The more electronegative the H373 imidazole group (i.e. when it is neutral and interacting with D282) is, the stronger the interaction with the Q377 amido group must be. This residue can thus help to orient H373 in an optimal conformation towards L-lactate at the reactants. Nevertheless, from our knowledge, no variant of Q377 has been experimentally studied.

Furthermore, several residues interact with the isoalloxazine ring: the K349 ammonium group with FMN N1 and the ribityl 2'-OH, the S228 hydroxyl group with FMN O4, the Q252 side chain oxygen atom with FMN N3H and the T280 hydroxyl with FMN O2. Electrostatic interaction energies between these residues and the active site for our different systems are summarized in Table S2. The interaction with S228 is quite modest and generally increases when the FMN is reduced. For WT and Y254L variant, it is however stronger at TS, suggesting that the proximity of L-lactate and its negative charge also influence this interaction. The Q252 and T280 electrostatic interactions with the QM part do not present any modification during the reaction and remain lower than $12 \mathrm{kcal} / \mathrm{mol}$. In contrast, K349 strongly interacts with the active site. The values reported in Table S2 correspond to the electrostatic interactions between the MM residue and the whole QM part. The eventual increase of attractive interactions between the reduced FMN and its neighbours can be balanced by the increase of repulsive interactions with the positively charged H373.

\section{Conclusions}

In this work, we analysed in the Fcb2 L-lactate oxidation mechanism in the context of the hydride transfer hypothesis. We have presented an extensive computational study combining simulations in WT and mutated enzymes. Our results provide a comprehensive molecular description of the hydride mechanism and the role of some active site residues. 
Our approach consists in DFT corrected AM1/MM MD to obtain free energy surfaces of the reaction consisting in proton and hydride transfers from L-lactate to H373 and FMN respectively. We have applied this methodology to determine the most catalytically efficient geometry of the active site arginine R289 and the effect of two mutations: Y254L and D282N.

It appears that an efficient reaction pathway in the WT enzyme consists in an asynchronous mechanism with first proton and then hydride transfer to respectively $\mathrm{H} 373 \mathrm{~N} \varepsilon$ and FMN N5. A shallow intermediate state is found in our 2D-PMF. A second pathway can be encountered, proceeding by concerted proton and hydride transfers. When R289 is kept in the proximal position, the free energy surface describes only this second pathway, with a free energy of activation higher than the one computed for R289 in a distal position (more than $12 \mathrm{kcal} / \mathrm{mol}$ ). Increasing interactions between the substrate carboxylate and the R289 side chain lead to three complementary effects: $i$ ) a decrease of steric hindrance around the lactate $\alpha \mathrm{C}$ substituents; ii) a loss of interaction between the lactate hydroxyl group and H373 and Y254; and iii) an increase of the electrophilic character of $\alpha \mathrm{C}$. The hydride transfer mechanism thus requires $\mathrm{R} 289$ in a distal position, maintained by an interaction with D292 and a water molecule but a proximal position of this residue must probably be more efficient in the carbanion mechanism. This assertion has to be confirmed by further theoretical studies of the carbanion mechanism; the first ones show that $\alpha \mathrm{H}$ departure to $\mathrm{H} 373$ is easier with the $\mathrm{R} 289$ proximal conformation (unpublished results).

Y254L and D282N variants also oxidize L-lactate following a concerted pathway. In these systems, hydride departure clearly precedes proton transfer. In these two variants, the product of the hydroxyl proton transfer alone is not as well stabilized as in the WT enzyme. By interacting with the deprotonated substrate alcohol, or with the positive H373 imidazolium group, Y254 and D282 respectively permit the hydroxyl proton transfer to H373 which decreases the hydride transfer free energy of activation. In their absence, the proton transfer cannot occur before $\alpha \mathrm{C}-\alpha \mathrm{H}$ bond breaking or weakening. $\alpha \mathrm{H}$ departure from lactate could allow electron transfer to FMN. Then, a negative charge on the isoalloxazine ring could help to stabilize the positively charged histidine and induce the proton transfer from lactate to H373. In the Y254L variant, a loss of steric hindrance around the substrate also makes the catalytic conformation of the substrate more difficult to reach, as observed for R289 in the proximal position. This geometrical effect could explain the difference between the experimental rate constants for the 
Y254L variant and the Y254F or the D282N variants; the latter only affect the electrostatic force field in the active site.

\section{$\underline{\text { References }}$}

1 F. Lederer, in Chemistry and biochemistry of flavoenzymes, Müller, F, Ed, Boca Raton, CRC Press., 1991, vol. 2, pp. 153-242.

2 S. Ghisla and V. Massey, Eur. J. Biochem., 1989, 181, 1-17.

3 L. M. Cunane, J. D. Barton, Z.-W. Chen, F. E. Welsh, S. K. Chapman, G. A. Reid and F. S. Mathews, Biochemistry, 2002, 41, 4264-4272.

4 W. Humphrey, A. Dalke and K. Schulten, J. Mol. Graph., 1996, 14, 33-38.

5 Z. Xia and F. S. Mathews, J. Mol. Biol., 1990, 212, 837-863.

6 M. Tegoni and C. Cambillau, Protein Sci., 1994, 3, 303-313.

7 C. G. Mowat, I. Beaudoin, R. C. E. Durley, J. D. Barton, A. D. Pike, Z. Chen, G. A. Reid, S. K. Chapman, F. S. Mathews and F. Lederer, Biochemistry, 2000, 39, 3266-3275.

8 G. A. Reid, S. White, M. T. Black, F. Lederer, F. S. Mathews and S. K. Chapman, Eur. J. Biochem., 1988, 178, 329-333.

9 R. Ghrir and F. Lederer, Eur. J. Biochem., 1981, 120, 279-282.

10P. F. Fitzpatrick, Acc. Chem. Res., 2001, 34, 299-307.

11 P. F. Fitzpatrick, Bioorganic Chem., 2004, 32, 125-139.

12P. Urban and F. Lederer, J. Biol. Chem., 1985, 260, 11115-11122.

13 K. S. Rao and F. Lederer, Protein Sci., 1998, 7, 1531-1537.

14T. C. Bruice, Acc. Chem. Res., 1980, 13, 256-262.

15 A. R. Dewanti and B. Mitra, Biochemistry, 2003, 42, 12893-12901.

16F. Lederer, A. Belmouden and M. Gondry, Biochem. Soc. Trans., 1996, 24, 77-83.

17 M. Gondry, J. Dubois, M. Terrier and F. Lederer, Eur. J. Biochem., 2001, 268, 4918-4927.

18F. Lederer, D. Amar, A. K. Ould Boubacar and C. Vignaud, in Flavins and Flavoproteins

2005, Nishino, T., Miura, R., Tanokura, M., and Fukui, K., Eds., Tokyo, ArchiTect Inc., 2005, pp. 193-204.

19J. Dubois, S. K. Chapman, F. S. Mathews, G. A. Reid and F. Lederer, Biochemistry, 1990, 29, 6393-6400.

20 P. Sobrado and P. F. Fitzpatrick, Biochemistry, 2003, 42, 15208-15214.

$21 \mathrm{M}$. Gondry and F. Lederer, Biochemistry, 1996, 35, 8587-8594.

22 S. Daff, F. D. Manson, G. A. Reid and S. K. Chapman, Biochem J, 1994, 301, 829-834.

23 R. Sinclair, G. A. Reid and S. K. Chapman, Biochem. J., 1998, 333, 117-120.

24C. G. Mowat, A. Wehenkel, A. J. Green, M. D. Walkinshaw, G. A. Reid and S. K. Chapman, Biochemistry, 2004, 43, 9519-9526.

25 D. Pompon, M. Iwatsubo and F. Lederer, Eur. J. Biochem., 1980, 104, 479-488.

26P. Sobrado, S. C. Daubner and P. F. Fitzpatrick, Biochemistry, 2001, 40, 994-1001.

27P. Macheroux, S. Ghisla, C. Sanner, H. Rüterjans and F. Müller, BMC Biochem., 2005, 6, 26.

28 G. Tabacchi, D. Zucchini, G. Caprini, A. Gamba, F. Lederer, M. A. Vanoni and E. Fois, FEBS

J., 2009, 276, 2368-2380.

29E. A. Carter, G. Ciccotti, J. T. Hynes and R. Kapral, Chem. Phys. Lett., 1989, 156, $472-477$.

30 R. Car and M. Parrinello, Phys. Rev. Lett., 1985, 55, 2471-2474. 
31 O. Dmitrenko, C. Thorpe and R. D. Bach, J. Phys. Chem. B, 2003, 107, 13229-13236.

32 M. J. Field, M. Albe, C. Bret, F. Proust-De Martin and A. Thomas, J. Comput. Chem., 2000,

21, 1088-1100.

33 C. R. Søndergaard, M. H. M. Olsson, M. Rostkowski and J. H. Jensen, J. Chem. Theory

Comput., 2011, 7, 2284-2295.

34 M. H. M. Olsson, C. R. Søndergaard, M. Rostkowski and J. H. Jensen, J. Chem. Theory

Comput., 2011, 7, 525-537.

35 M. J. S. Dewar, E. G. Zoebisch, E. F. Healy and J. J. P. Stewart, J. Am. Chem. Soc., 1985, 107, 3902-3909.

36 M. J. Field, P. A. Bash and M. Karplus, J. Comput. Chem., 1990, 11, 700-733.

37 W. L. Jorgensen, D. S. Maxwell and J. Tirado-Rives, J. Am. Chem. Soc., 1996, 118, $11225-$ 11236.

38 W. L. Jorgensen, J. Chandrasekhar, J. D. Madura, R. W. Impey and M. L. Klein, J. Chem.

Phys., 1983, 79, 926-935.

39 G. M. Torrie and J. P. Valleau, J. Comput. Phys., 1977, 23, 187-199.

40 S. Kumar, J. M. Rosenberg, D. Bouzida, R. H. Swendsen and P. A. Kollman, J. Comput.

Chem., 1992, 13, 1011-1021.

41 J. J. Ruiz-Pernía, E. Silla, I. Tuñón, S. Martí and V. Moliner, J. Phys. Chem. B, 2004, 108, 8427-8433.

42 J. J. Ruiz-Pernía, E. Silla, I. Tuñón and S. Martí, J. Phys. Chem. B, 2006, 110, 17663-17670.

43 J. C. Corchado, E. L. Coitiño, Y.-Y. Chuang, P. L. Fast and D. G. Truhlar, J. Phys. Chem. A, 1998, 102, 2424-2438.

44 Y.-Y. Chuang, J. C. Corchado and D. G. Truhlar, J. Phys. Chem. A, 1999, 103, 1140-1149.

45 K. A. Nguyen, I. Rossi and D. G. Truhlar, J. Chem. Phys., 1995, 103, 5522.

46R. J. Renka, SIAM J. Sci. Stat. Comput., 2006.

47 R. J. Renka, ACM Trans Math Softw, 1993, 19, 81-94.

48 Y. Zhao and D. G. Truhlar, Theor. Chem. Acc., 2008, 120, 215-241.

49G. A. Petersson, A. Bennett, T. G. Tensfeldt, M. A. Al-Laham, W. A. Shirley and J. Mantzaris, J. Chem. Phys., 1988, 89, 2193-2218.

50 G. A. Petersson and M. A. Al-Laham, J. Chem. Phys., 1991, 94, 6081-6090.

51 M. J. Frisch, G. W. Trucks, H. B. Schlegel, G. E. Scuseria, M. A. Robb, J. R. Cheeseman, G.

Scalmani, V. Barone, B. Mennucci, G. A. Petersson, H. Nakatsuji, M. Caricato, X. Li, H. P.

Hratchian, A. F. Izmaylov, J. Bloino, G. Zheng, J. L. Sonnenberg, M. Hada, M. Ehara, K.

Toyota, R. Fukuda, J. Hasegawa, M. Ishida, T. Nakajima, Y. Honda, O. Kitao, H. Nakai, T.

Vreven, J. A. Montgomery Jr., J. E. Peralta, F. Ogliaro, M. J. Bearpark, J. Heyd, E. N.

Brothers, K. N. Kudin, V. N. Staroverov, R. Kobayashi, J. Normand, K. Raghavachari, A. P.

Rendell, J. C. Burant, S. S. Iyengar, J. Tomasi, M. Cossi, N. Rega, N. J. Millam, M. Klene, J.

E. Knox, J. B. Cross, V. Bakken, C. Adamo, J. Jaramillo, R. Gomperts, R. E. Stratmann, O.

Yazyev, A. J. Austin, R. Cammi, C. Pomelli, J. W. Ochterski, R. L. Martin, K. Morokuma, V.

G. Zakrzewski, G. A. Voth, P. Salvador, J. J. Dannenberg, S. Dapprich, A. D. Daniels, Ö.

Farkas, J. B. Foresman, J. V. Ortiz, J. Cioslowski and D. J. Fox, Gaussian 09, Gaussian, Inc.,

Wallingford, CT, USA, 2009.

52 K. Świderek, I. Tuñón, V. Moliner and J. Bertran, ACS Catal., 2015, 5, 2587-2595.

53 K. Świderek, A. Kohen and V. Moliner, Phys. Chem. Chem. Phys., 2015, 17, 30793-30804.

54 S. Ferrer, J. J. Ruiz-Pernía, I. Tuñón, V. Moliner, M. Garcia-Viloca, A. González-Lafont and J.

M. Lluch, J. Chem. Theory Comput., 2005, 1, 750-761.

55 K. Świderek, I. Tuñón, S. Martí and V. Moliner, ACS Catal., 2015, 5, 1172-1185. 
56 Y. Cao, S. Han, L. Yu, H. Qian and J.-Z. Chen, J. Phys. Chem. B, 2014, 118, 5406-5417.

57F. Lederer, Protein Sci., 1992, 1, 540-548.

58 N. Rouvière-Fourmy, C. Capeillère-Blandin and F. Lederer, Biochemistry, 1994, 33, 798-806.

59C. S. Miles, N. Rouvière-Fourmy, F. Lederer, F. S. Mathews, G. A. Reid, M. T. Black and S. K. Chapman, Biochem J, 1992, 285, 187-192. 